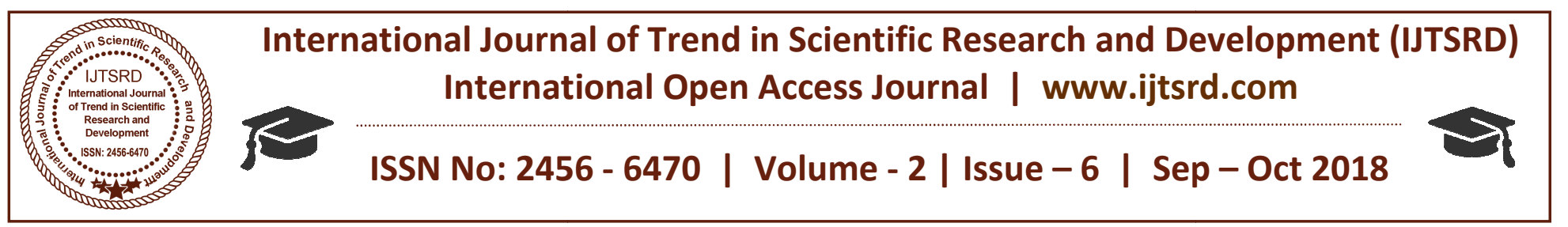

\title{
Solving Initial-Value Problem of the First-Order Differential Equation by Euler's Method using Casio fx 570EX Classwiz Scientific Calculator
}

\author{
K. G. Tay \\ Associate professor, \\ Communication Department, \\ Faculty of Electrical and \\ Electronic, University Tun \\ Hussein Onn, Malaysia
}

\author{
T. H. Cheong \\ Lecturer, Faculty of Education, \\ University Technology Mara, \\ Malaysia
}

\author{
W. K. Tiong \\ Senior Lecturer, Department of \\ Computational Science and \\ Mathematics, University of \\ Malaysia, Sarawak, Malaysia
}

\section{ABSTRACT}

Solving numerical methods manually without using a scientific calculator or using scientific calculator traditionally without input the iterative formula into the calculator can be quite tedious and boring due to its repetitive calculations. There is a series of studies discussing the implementation of numerical methods using Excel spread sheet as well as Casio scientific calculators, such as Casio fx-570 MS scientific calculator, Casio fx-570 ES scientific calculator and Casio fx-570 ES plus scientific calculator. Even though Excel spreadsheet made the implementation of numerical methods easier to be understood by the new learner of numerical methods, but it is not portable for classrooms (teaching and learning purposes) and examination hall (evaluation purpose). All the three mentioned models of scientific calculators allow the input of iterative formula but users still need to rein put the new inputs then press CALC button to get its iterative solutions. The new model of Casio fx-570 FX classwiz scientific calculator offers a spreadsheet ability of 45 rows and 5 columns which made the implementation of the numerical methods much easier if compared to previous models. Hence, in this paper, we solved the initial-value problem (IVP) of the firstorder ordinary differential equations by the Euler's method using Casio fX-570 FX classwiz scientific calculator for the classroom and examination purposes.

Keyword: IVP, ODE, Euler, Casio

\section{INTRODUCTION}

An ordinary differential equation (ODE) is a differential equation consists of the functions of only one independent variable and its normal derivatives. ODEs arise in many physical problems including engineering, physics, economics, biology and etc. The order of an ODE is determined by its highest derivatives. Hence, there are first-order, second-order and higher-order QDEs.

When the analytical solution of an ODE is not available, we have to seek its approximate solution by using numerical methods. There are several numerical methods such as the Euler's, Taylor series, midpoint, Heun, fourth-order Runge-Kutta (RK4) methods and etc to solve an IVP of the first-order ODE.

Solving numerical methods manually without using a scientific calculator or using scientific calculator traditionally without input the formula into the calculator can be quite tedious and boring due to its repetitivecalculations. Hencethere is a series of books discussing the implementation of numerical methods using Casio scientific calculator, such as Casio fx-570 MS scientific calculator in [1], Casio fx-570 ES scientific calculator in [2] and Casio fx-570 ES plus scientific calculator in [3].

The studies on solving numerical methods in classroom and examination situations using a calculator, focusing on nonlinear equations [4]-[5], systems of linear equations [6], interpolation [7], an approximation of integration [8], and computing of eigen values [9]-[10]. 
Recently, there is a new model of scientific calculator which is Casio fx-570EX classwiz which offers the spreadsheet ability. It provides 45 rows and 5 columns of spreadsheet ability and functions almost same with Excel spreadsheet. For the classroom and examination purposes, the usage of the scientific calculator will be appropriate if compared to Excel spreadsheet due to its portability. Hence, in this paper, we would like to solve the IVP of the firstorder ODE by Euler's method using Casiofx-570EX classwiz scientific calculator for the classroom and examination purposes.

INITIAL-VALUE PROBLEM (IVP) OF THE FIRST-ORDER DIFFERENTIAL EQUATIONS

Consider an IVP of the first-order differential equation

$$
y^{\prime}=f(x, y), \quad y\left(x_{0}\right)=y_{0}, \quad x_{0} \leq x \leq x_{n} .
$$

By using numerical methods, the interval of $x$ is divided into $n$ subintervals with step size $h$ such that $x_{i}=x_{0}+i h$. we need to solve $y$ from $i=1$ to $n$. It was noticed that by using numerical methods, we only obtained discrete values of $y$. However, if we solved the differential equation analytically, we obtained a continuous function of $y(x)$. The Euler is discussed here.

\section{Euler's Method}

The solution of Eq. (1) by Euler's method is given by

$$
y_{i+1}=y_{i}+h y_{i}^{\prime}=y_{i}+h f\left(x_{i}, y_{i}\right),
$$

Where $h$ is a step size.

\section{NUMERICAL EXAMPLES}

The $R C$ series circuit can be described by the following differential equation

$$
R C \frac{d V}{d t}+V=E(t)
$$

With initial condition $V(0)=0$. If $R=10 \Omega, C=$ $0.004 \mathrm{~F}$, and $E=85 \cos (150 \mathrm{t}) \mathrm{V}$, the voltage $V$ for $0 \leq t \leq 0.05$ in the $R C$ series circuit was found by using the Euler's method with $\Delta t=0.01$. The absolute errors were found if the exact solution is given by

$$
V=\frac{510}{37} \sin 150 t+\frac{85}{37}\left(\cos (150 t)-e^{-25 t}\right)
$$

\section{Solution by Euler's Method}

Step 1: By substituting $R=10 \Omega, C=0.004 \mathrm{~F}$, and $E$ $=85 \cos (150 \mathrm{t}) \mathrm{V}$ into the first-order differential equation, we obtained

$$
\begin{gathered}
10(0.004) \frac{d V}{d t}+V=85 \cos (150 t) \\
\frac{d V}{d t}=2125 \cos (150 t)-25 V=f(t, V)
\end{gathered}
$$

Step 2: The solution by Euler's method is given by

$$
\begin{aligned}
V_{k+1} & =V_{k}+h f(t, V) \\
& =V_{k}+0.01\left(2125 \cos (150 t)-25 V_{k}\right)
\end{aligned}
$$

Where $h=\Delta t=0.01$.

Step 3: By using Spreadsheet mode 8 in the calculator, tabulating $t$ values in column A, filling initial condition in cell B1 as zero and pressing OPTN button will give

\section{$1:$ Fil 1 Formula \\ 2:Fill Value \\ $3:$ Edit Cell \\ 4:Free Space}

Choose 1 will get

\begin{tabular}{|ll|}
\hline Fi 11 & Formula \\
Form & $=$ \\
Range & : B2: B2 \\
\hline
\end{tabular}

Type in the following formula:

$$
=B 1+0.01(2125 \cos (150 A 1)-25 B 1)
$$

Then press =button, will yield

\begin{tabular}{|l|} 
Fill Formula \\
Form $=B 1+0.01(21$ \\
Range $: B 2: B 2$
\end{tabular}

Next,choose to fill in the formula from cells B2: B6 as follows:

Fill Formula
Form $=$ B $1+0.01(21$
Range $:$ B2:B6


To key in the variables A, B, C, D, E, F, X, Y and M in the calculator users have to press Alpha button then follow by the variable which is displayed as red colour on top of the main buttons. The approximate $V$ values are displayed in column B

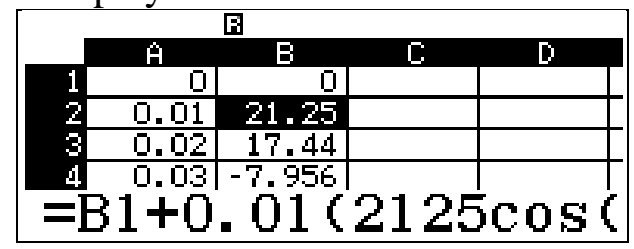

Step 4: Pressing OPTN button to fill in exact solution formula in cell $\mathrm{C} 1$ as below:

$$
=\frac{510}{37} \sin (150 A 1)+\frac{85}{37}\left(\cos (150 A 1)-e^{-25 A 1}\right)
$$

Then choose to fill in the formula from cells C1: C6 will results in:

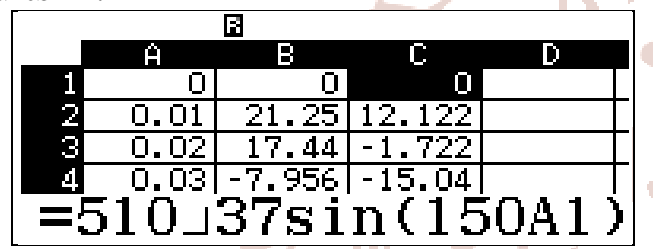

Step 5 Pressing OPTN button to fill in absolute error formula in cell $\mathrm{D} 1$ as below:

$$
=A b s(B 1-C 1)
$$

Then choose to fill in the formula from cells D1: D6 will display:

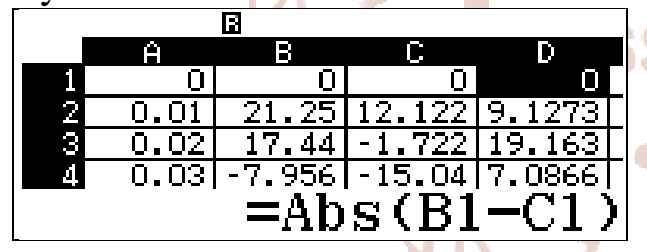

The complete solution is shown in Table 1 below:

TABLE1. The solution

\begin{tabular}{|c|c|c|c|c|}
\hline $\mathrm{k}$ & $\mathrm{t}$ & $\mathrm{V}$ & Exact & |Error| \\
\hline 0 & 0 & 0 & 0 & 0 \\
\hline 1 & 0.01 & 21.25 & 12.122 & 9.1273 \\
\hline 2 & 0.02 & 17.44 & -1.722 & 19.163 \\
\hline 3 & 0.03 & -7.956 & -15.04 & 7.0866 \\
\hline 4 & 0.04 & -10.44 & -2.49 & 7.9563 \\
\hline 5 & 0.05 & 12.568 & 13.067 & 0.4989 \\
\hline
\end{tabular}

\section{CONCLUSION}

We have solved the IVP of the first-order ODE by Euler's method using spreadsheet mode in Casio fx-
570EX classwiz scientific calculator for the classroom and examination purposes. It is easier and faster than the previous models such as Casio fx-570 MS, Casio fx-570 ES and Casio fx-570 ES Plus. The procedure presented here can be applied to second-order Taylor series method, midpoint method and Heun's method but not the fourth-order Runge-Kutta(RK4) method due to the number of column sin Casio fx-570EX classwiz scientific calculator is limited to five. To solve the IVP of the first-order ODE by using theRK4 method, five columns are needed. Even though Excel spreadsheet will be easier to work around (larger rows and columns, bigger view, easier to scroll around, move, copy and more abilities) if compared to spreadsheet mode in Casio fx-570EX classwiz scientific calculator, but students can only bring scientific calculator for classroom and examination purposes, hence spreadsheet mode in Casio fx-570EX classwiz offers a portable spreadsheet environment to implement numerical methods easily if compared to old version of calculator.

\section{REFERENCES}

1. K. G. Tay. How To Use Calculator Casio Fx$570 \mathrm{~ms}$ In Numerical Methods (PenerbitKUiTTHO, BatuPahat, 2006).

2. K. G. Tay, K. B. Lim andR. Abdul-Kahar. Numerical. MethodsUsing Casio Fx-570ES Calculator (PenerbitUTHM, BatuPahat, 2011).

3. K. G. Tay, C. Phangand K. B. Lim. Numerical. MethodsWith Casio Fx-570 ES Plus Calculator (PenerbitUTHM, BatuPahat, 2017).

4. K. B. Lim, G. J. Yong, T. H. Cheong and K. G. Tay,"The Effectiveness of Using Calculator in Solving Non-Linear Equations by NewtonRaphson Method" Proc. of the 16th Asian Tech. Conf. Math. Turkey, 2011, pp. 291 - 297.

5. T. H. Cheong, K. B. Lim, and K. G. Tay,"Solving Non-Linear Equation by Newton-Raphson Method using Buit-in Derivative Function in Casio fx-570Es Calculator"Proc. of the 16th Asian Tech. Conf. Math. Turkey, 2011, pp. 307-316.

6. R. Abdul Kahar, K. G. Tay and K. B. Lim,"Solving System Of Linear Equations With Gauss-Seidel Iteration Method Using Casio Fx570es Calculator" Proceeding of the National Symposium on Application of Science Mathematics 2010 (SKASM 2010) and 18th Mathematical Science National Symposium (SKSM 2010). Johor Bahru, 2010, pp. 397-403. 
International Journal of Trend in Scientific Research and Development (IJTSRD) ISSN: 2456-6470

7. T. H. Cheong and K. G. Tay, AIP Conference Proceedings.1775, 1-5.(2016).

8. K. B. Lim, K. G., Tay and R. Abdul Kahar, "Using Scientific Calculator on Trapezoidal Rule" Proceeding of the National Symposium on Application of Science Mathematics 2010 (SKASM 2010) and 18th Mathematical Science National Symposium (SKSM 2010). Johor Bahru, 2010, pp. 425-429.

9. R. Abdul Kahar and K. G. Tay, "Casio fx-570ES Calculator for Approximating the Dominant Eigen value Using Power Method" National Seminar on Science and Technology 2009 (PKPST 2009). BatuPahat, 2009, pp. 255-259.

10. R. Abdul Kahar, and K. G. Tay,"Utilizing Casio fx-570ES Calculator to Approximate The Smallest Eigen value Using Inverse Power Method" Proceeding of the 17th National Symposium on Mathematical Science (SKSM 2009). Malacca, 2009, pp.1069-1073. (2009).

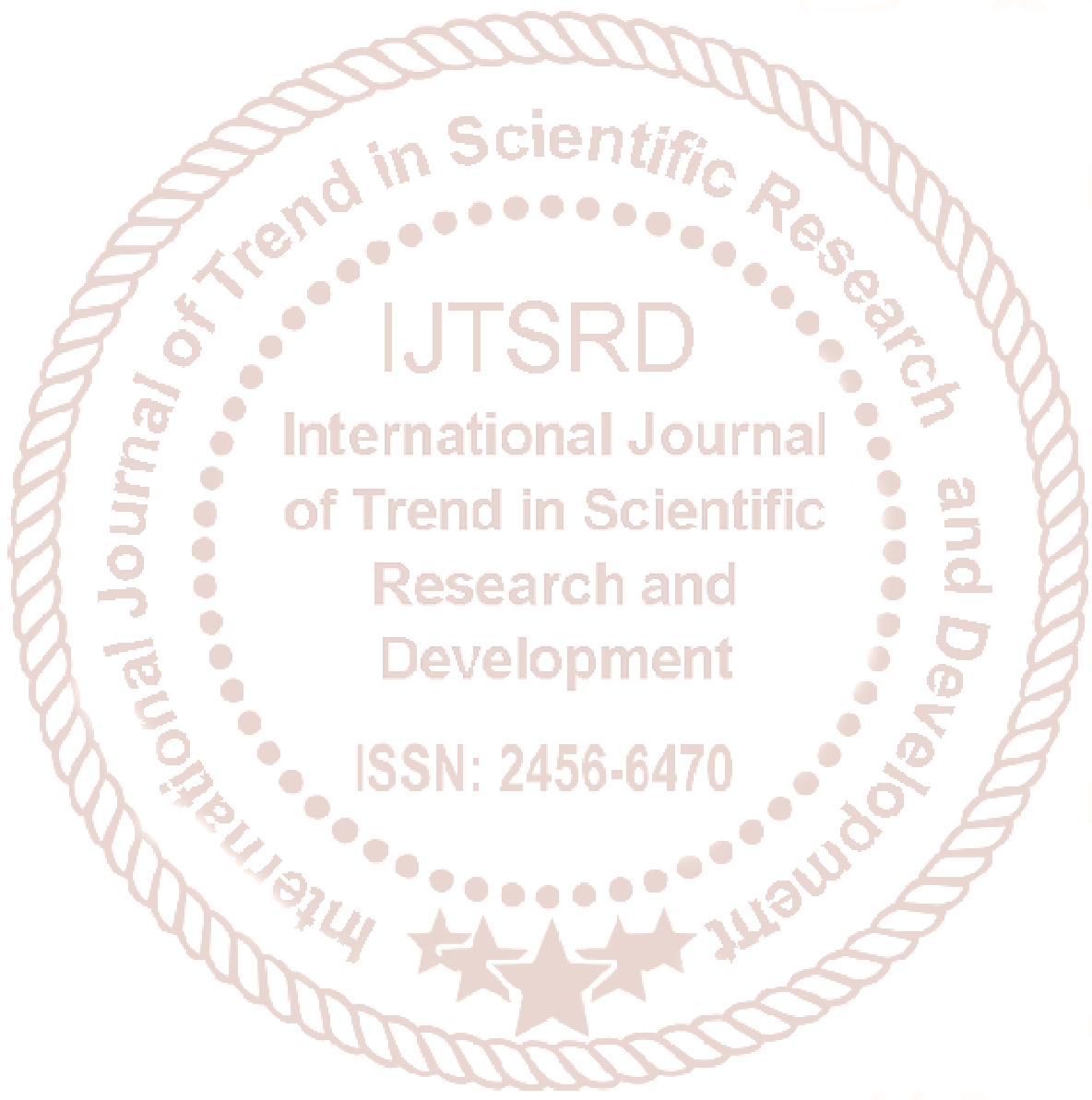

